\title{
DOA estimation for conformal vector- sensor array using geometric algebra
}

\author{
Tianzhen Meng, Minjie Wu ${ }^{*}$ and Naichang Yuan
}

\begin{abstract}
In this paper, the problem of direction of arrival (DOA) estimation is considered in the case of multiple polarized signals impinging on the conformal electromagnetic vector-sensor array (CVA). We focus on modeling the manifold holistically by a new mathematical tool called geometric algebra. Compared with existing methods, the presented one has two main advantages. Firstly, it acquires higher resolution by preserving the orthogonality of the signal components. Secondly, it avoids the cumbersome matrix operations while performing the coordinate transformations, and therefore, has a much lower computational complexity. Simulation results are provided to demonstrate the effectiveness of the proposed algorithm.
\end{abstract}

Keywords: Geometric algebra, Conformal array, Electromagnetic vector sensors, DOA estimation

\section{Introduction}

The direction of arrival (DOA) estimation has received a strong interest in wireless communication systems such as radar, sonar, and mobile systems [1]. In this correspondence, the problem of DOA estimation is considered in the case of multiple polarized signals impinging on the conformal vector-sensor array (CVA). We name our target array CVA since it is a conformal array whose elements are electromagnetic vector sensors. Interest in this problem can be divided into two topics: (1) conformal array and (2) electromagnetic vector sensors.

A conformal antenna is an antenna that conforms to a prescribed shape. The shape can be some part of an airplane, high-speed missile, or other vehicle [2]. Their benefits include reducing aerodynamic drag, covering wide angle, space-saving and so on [3, 4]. Nevertheless, due to the curvature of the bearing surface, the far-field contribution in the incident direction of one element is different from that of others [5]. The pattern synthesis theorem is not available resulting from the fact that the conformal arrays can no longer be regarded as simple isotropic ones. In [4], Wang et al. proposed a uniform method for the element-polarized pattern transformation of arbitrary three-dimensional (3-D) conformal arrays based on Euler rotation. However, the Euler

\footnotetext{
* Correspondence: wmj601@nuaa.edu.cn

College of Electronic Science and Engineering, National University of Defense Technology, Deya Road 109, Changsha 410073, China
}

rotation involves cumbersome matrix transformations, and therefore, has a considerable computational burden. Zou et al. analyzed the 3-D pattern of arbitrary conformal arrays using geometric algebra in [6]. Nevertheless, this mathematical language was not transplanted to the DOA estimation. In view of this, Wu et al. combined the geometric algebra with multiple signal classification (MUSIC), termed as GA-MUSIC, to solve the DOAs for cylindrical conformal array [7]. It used short dipole as the element which made the array belong to a scalar array. In addition, the electromagnetic vector sensors are not taken into account.

As for the second point, we know the electromagnetic vector sensor can measure the three components of the electric field and the three components of the magnetic field simultaneously. And, considerable studies on the extensions of traditional array signal processing techniques to the vector sensors are available in literature. In [8], Nehorai concatenated all the output vectors into a long vector and derived the Cramer-Rao bound (CRB). However, the orthogonality of the signal components was lost in this case. In view of this, a hypercomplex model for multicomponent signals impinging on vector sensors was presented in [9]. This model was based on biquaternions (quaternions with complex coefficients). Subsequently, Jiang et al. introduced geometric algebra into the electromagnetic vector-sensor processing field [10]. However, the model cannot be applied to the 
conformal array since the pattern is assumed to be a scalar and the same for each element.

In this correspondence, we will combine the electromagnetic vector sensors with the conformal array, and present a unified model based on geometric algebra to estimate the DOAs. The proposed technique in this paper is regarded as a generalization of the one presented in [10] to the case of the conformal arrays. Compared with existing methods, the proposed one has two main advantages. Firstly, it can give a more accurate estimation by preserving the orthogonality of the signal components. Secondly, it largely decreases the computation complexity for the coordinate transformations are avoided. In addition, it has a strong commonality, that is to say, it is not limited to any specific conformal array.

The rest of this paper is as follows. In Section 2, some notations about geometric algebra are briefly introduced, and on this basis, the manifold for the conformal vectorsensor array is derived. Section 3 analyzes the computational burden. Illustrative examples are carried out to verify the effectiveness of the proposed algorithm in Section 4 , followed by concluding remarks.

Throughout this correspondence, we use lowercase boldface letters to denote vectors and uppercase boldface letters to represent matrices for notational convenience. Moreover, the uppercase letters symbolize the multivectors whenever there is no possibility of confusion. Superscripts “"”, " $T$ ", and " $H$ " represent the conjugation, transpose, and conjugate transpose, respectively. In addition, $(.)^{+}$and $(.)^{\sim}$ symbolize the conjugate transpose in geometric algebra and the reverse operator, respectively. Finally, $\Re_{3}^{m n}$ stands for the $m \times n$ real matrix in 3-D space and $E\{\cdot\}$ denotes the expectation operator.

\section{The proposed algorithm}

\subsection{Some notations about geometric algebra}

Geometric algebra is the largest possible associative algebra that integrates all algebraic systems (algebra of complex numbers, matrix algebra, quaternion algebra, etc.) into a coherent mathematical language [11]. In view of its widespread usage in subsequent sections, it is worthwhile to review some notations about geometric algebra before proceeding to the physical problems of interest.

The geometric product is considered as the fundamental product of geometric algebra, and its definition is as follows

$$
x y=x \cdot y+x \wedge y
$$

where the wedge symbol " " denotes the outer product with the properties listed in Table 1.

Exchanging the order of $\boldsymbol{x}$ and $\boldsymbol{y}$ in (1),and utilizing the symmetry of the inner product and the antisymmetry of the outer product, it follows that
Table 1 Properties of the outer product

\begin{tabular}{ll}
\hline Property & Meaning \\
\hline Anti-symmetry & $(\boldsymbol{x} \wedge \boldsymbol{y})=-(\boldsymbol{y} \wedge \boldsymbol{x})$ \\
Scaling & $\boldsymbol{x} \wedge(\gamma \boldsymbol{y})=\gamma(\boldsymbol{x} \wedge \boldsymbol{y})$ \\
Distributivity & $\boldsymbol{x} \wedge(\boldsymbol{y}+\boldsymbol{z})=(\boldsymbol{x} \wedge \boldsymbol{y})+(\boldsymbol{x} \wedge \boldsymbol{z})$ \\
Associativity & $\boldsymbol{x} \wedge(\boldsymbol{y} \wedge \boldsymbol{z})=(\boldsymbol{x} \wedge \boldsymbol{y}) \wedge \boldsymbol{z}$ \\
\hline
\end{tabular}

$$
y x=x \cdot y \cdot x \wedge y
$$

Combining (1) with (2), we can find that the inner product and the outer product can be uniformly represented by the geometric product, that is,

$$
\begin{aligned}
& x \cdot y=\frac{x y+y x}{2} \\
& x \wedge y=\frac{x y-y x}{2}
\end{aligned}
$$

In general, we call an outer product of $k$ vectors a $k$ blade. The value of $k$ is referred to as the grade of the blade. Specially, the top-grade blade $\boldsymbol{E}_{n}$ in an $n$-dimensional space is called pseudo-scalars. Essentially, blades are just elements of the geometric algebra. It is noted that we restrict the discussion to 3-D Euclidean space [12], that is, a space with an orthonormal basis $\left\{\boldsymbol{e}_{x}, \boldsymbol{e}_{y}\right.$, $\left.\boldsymbol{e}_{z}\right\}$. As shown in Fig. 1, $\boldsymbol{E}_{3}$ is the pseudo-scalar, relative to the origin denoted by $\boldsymbol{O}$. The three-blade is drawn as a parallelepiped. The volume depicts the weight of the three-blade. Nevertheless, blades have no specific shape.

A linear combination of blades with different grades is called a multivector [13]. Multivectors are the general elements of geometric algebra. Thus, a generic element can be expressed by

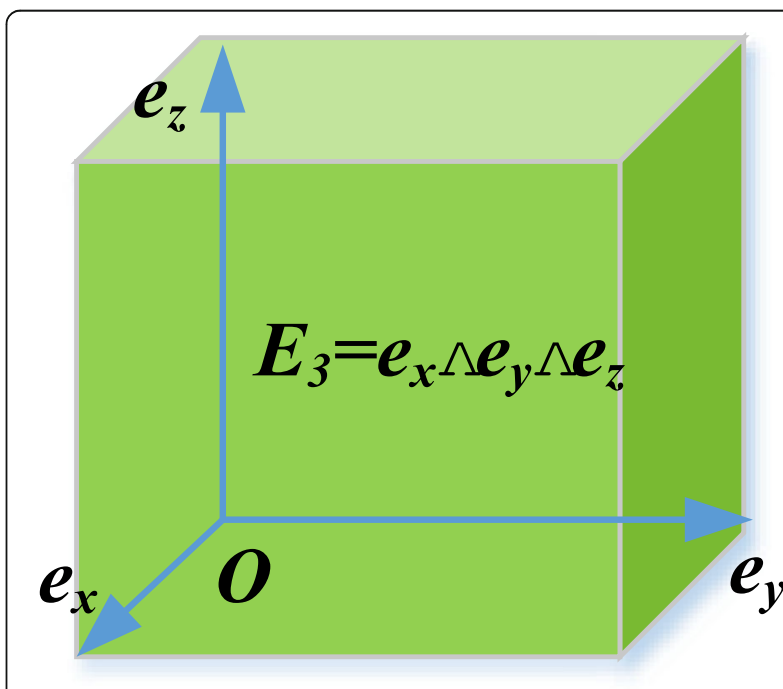

Fig. 1 The geometry of 3-blade 


$$
\begin{aligned}
A= & a_{0}+a_{1} \boldsymbol{e}_{x} \wedge \boldsymbol{e}_{y}+a_{2} \boldsymbol{e}_{z} \wedge \boldsymbol{e}_{x}+a_{3} \boldsymbol{e}_{y} \wedge \boldsymbol{e}_{z} \\
& +a_{4} \boldsymbol{e}_{x} \wedge \boldsymbol{e}_{y} \wedge \boldsymbol{e}_{z}+a_{5} \boldsymbol{e}_{z}+a_{6} \boldsymbol{e}_{y}+a_{7} \boldsymbol{e}_{x}
\end{aligned}
$$

where $a_{0}, a_{1}, \ldots, a_{7}$ are real numbers. For $\boldsymbol{e}_{x}, \boldsymbol{e}_{y}, \boldsymbol{e}_{z}$ are mutually orthogonal, using the definition of the geometric product, (5) can be represented by another shape.

$$
\begin{aligned}
A & =a_{0}+a_{1} \boldsymbol{e}_{x y}+a_{2} \boldsymbol{e}_{z x}+a_{3} \boldsymbol{e}_{y z}+a_{4} \boldsymbol{e}_{x y z}+a_{5} \boldsymbol{e}_{z}+a_{6} \boldsymbol{e}_{y}+a_{7} \boldsymbol{e}_{x} \\
& =\langle A\rangle_{0}+\langle A\rangle_{1}+\langle A\rangle_{2}+\langle A\rangle_{3}
\end{aligned}
$$

where the notation $\langle A\rangle_{k}$ means to select or extract the grade $k$ part of $A$ and the reverse of $\langle A\rangle_{k}$ can be calculated as follows

$$
\left\langle A^{\sim}\right\rangle_{k}=(-1)^{\frac{k(k-1)}{2}}\langle A\rangle_{k}
$$

Thus, the reverse of $A$ is given

$$
\widetilde{A}=\langle A\rangle_{0}+\langle A\rangle_{1}-\langle A\rangle_{2}-\langle A\rangle_{3}
$$

In the discussion up to this point, we can define the norm of a multivector.

$$
\|A\|=\sqrt{\left\langle A A^{\sim}\right\rangle_{0}}=\sqrt{\sum_{k=0}^{3}\left\langle\langle A\rangle_{k}\langle A\rangle_{k}\right\rangle_{0}}
$$

We will next introduce the rotor, one of the most important objects in applications of geometric algebra. As shown in Fig. 2, vector $y$ is acquired through rotating the vector $\boldsymbol{x}$ with $\theta$. The rotation can be regarded as two consecutive reflections, first in $\boldsymbol{a}$, then in $\boldsymbol{b}$. Correspondingly, the expression that reflects $x$ in the line with direction $\boldsymbol{a}$ is

$$
x^{\prime}=a x a=2(a \times x) a-x
$$

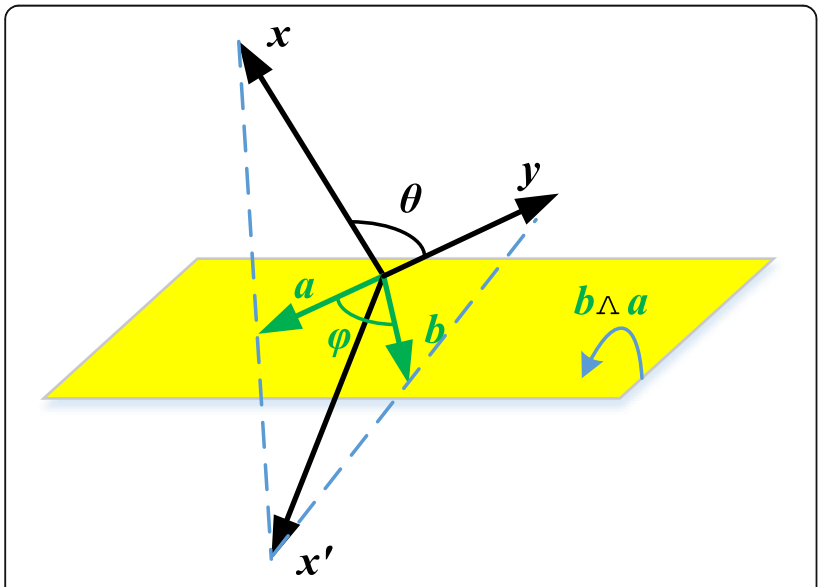

Fig. 2 Rotation of vector $\boldsymbol{x}$
The expression appears to be strange at first, but it is actually one of the most important rationales why the geometric product is so useful.

Similarly, $\boldsymbol{y}$ can be obtained by reflecting $\boldsymbol{x}^{\prime}$ in the line with direction $\boldsymbol{b}$

$$
\boldsymbol{y}=\boldsymbol{b} \boldsymbol{x}^{\prime} \boldsymbol{b}^{-1}=\boldsymbol{b a x} \boldsymbol{a}^{-1} \boldsymbol{b}^{-1}=(\boldsymbol{b a}) \boldsymbol{x}(\boldsymbol{b a})^{-1}=\boldsymbol{R} \boldsymbol{x} \boldsymbol{R}^{-1}
$$

As shown in Eq. (11), $\boldsymbol{R}$ is identified as the rotor. If we want to rotate a vector counterclockwise by a specific angle, we only need to apply the rotor to the vector. And, the rotation must be over twice the angle between $\boldsymbol{a}$ and $\boldsymbol{b}$. In Appendix 1, a brief proof is given.

\subsection{Complex representation matrix (CRM)}

As stated above, we adopt multivector as a generic element of geometric algebra. However, the analysis of the mulitvector and its attendant theory are scarce. In view of this, we will introduce the CRM since the matrix theories are mature [14]. Similar to the multivector, we construct the matrix in geometric algebra, noted $G_{3}^{m n}$, as follows

$$
\boldsymbol{A}=\boldsymbol{A}_{0}+\boldsymbol{A}_{1} \boldsymbol{e}_{x y}+\boldsymbol{A}_{2} \boldsymbol{e}_{z x}+\boldsymbol{A}_{3} \boldsymbol{e}_{y z}+\boldsymbol{A}_{4} \boldsymbol{e}_{x y z}+\boldsymbol{A}_{5} \boldsymbol{e}_{z}+\boldsymbol{A}_{6} \boldsymbol{e}_{x}+\boldsymbol{A}_{7} \boldsymbol{e}_{x}
$$

where $\boldsymbol{A}_{0}, \boldsymbol{A}_{1}, \ldots, \boldsymbol{A}_{7} \in \Re_{3}^{m n}$. Thus, the CRM can be defined as

$$
\psi(\boldsymbol{A})=\left[\begin{array}{cc}
\boldsymbol{A}_{0}+\boldsymbol{A}_{4} \boldsymbol{e}_{x y z}+\boldsymbol{A}_{1} \boldsymbol{e}_{x y z}+\boldsymbol{A}_{5} & -\boldsymbol{A}_{2}+\boldsymbol{A}_{6} \boldsymbol{e}_{x y z}-\boldsymbol{A}_{3} \boldsymbol{e}_{x y z}-\boldsymbol{A}_{7} \\
\boldsymbol{A}_{2}-\boldsymbol{A}_{6} \boldsymbol{e}_{x y z}-\boldsymbol{A}_{3} \boldsymbol{e}_{x y z}-\boldsymbol{A}_{7} & \boldsymbol{A}_{0}+\boldsymbol{A}_{4} \boldsymbol{e}_{x y z}-\boldsymbol{A}_{1} \boldsymbol{e}_{x y z}-\boldsymbol{A}_{5}
\end{array}\right]
$$

Given a matrix $\boldsymbol{A} \in G_{3}^{m n}$ and its CRM $\psi(\boldsymbol{A})$, then the following equalities stand

$$
\begin{aligned}
& \boldsymbol{A}=\boldsymbol{P}_{2 m} \psi(\boldsymbol{A}) \boldsymbol{P}_{2 n}^{+} \\
& \psi(\boldsymbol{A})=\boldsymbol{Q}_{2 m}\left[\begin{array}{ll}
\boldsymbol{A} & \\
& \boldsymbol{A}
\end{array}\right] \boldsymbol{Q}_{2 n}
\end{aligned}
$$

where

$$
\begin{aligned}
& \boldsymbol{P}_{2 m}=\frac{1}{2}\left[\left(1+\boldsymbol{e}_{z}\right) \boldsymbol{I}_{m}\left(\boldsymbol{e}_{z x}-\boldsymbol{e}_{x}\right) \boldsymbol{I}_{m}\right] \in G_{3^{m \times 2 m}} \\
& \boldsymbol{Q}_{2 m}=\frac{1}{2}\left[\begin{array}{cc}
\left(1+\boldsymbol{e}_{z}\right) \boldsymbol{I}_{m} & \left(\boldsymbol{e}_{z x}-\boldsymbol{e}_{x}\right) \boldsymbol{I}_{m} \\
\left(-\boldsymbol{e}_{z x}-\boldsymbol{e}_{x}\right) \boldsymbol{I}_{m} & \left(1-\boldsymbol{e}_{z}\right) \boldsymbol{I}_{m}
\end{array}\right] \in G_{3^{2 m \times 2 m}}
\end{aligned}
$$

with $\boldsymbol{I}_{m}$ being the identity matrix of dimension $m \times m$. Properties (14) and (15) can be verified by direct calculation using Eq. (16) and Eq. (17). For $\boldsymbol{e}_{x y z}$ is isomorphic to complex imaginary unit $j$ [9], $\psi(\boldsymbol{A})$ can be regarded as a complex matrix. Then, all the operation rules of the complex matrix are applicable to $\psi(\boldsymbol{A})$. Some properties [15] which will be used in the sequel are listed as follows. 

a) $\boldsymbol{A}=\boldsymbol{B} \Leftrightarrow \psi(\boldsymbol{A})=\psi(\boldsymbol{B})$
b) $\psi(\boldsymbol{A}+\boldsymbol{B})=\psi(\boldsymbol{A})+\psi(\boldsymbol{B}), \psi(\boldsymbol{A C})=\psi(\boldsymbol{A}) \psi(\boldsymbol{C})$;
c) $\psi\left(\boldsymbol{A}^{+}\right)=\psi^{+}(\boldsymbol{A})$.

It is also worthwhile to note that the following three properties regarding $\boldsymbol{P}_{2 m}$ and $\boldsymbol{Q}_{2 m}$ will be of use in the forthcoming calculations.

d) $\boldsymbol{P}_{2 m} \boldsymbol{P}_{2 m}^{+}=\boldsymbol{I}_{m}$;

e) $\boldsymbol{P}_{2 m}^{+} \boldsymbol{P}_{2 m} \psi(\boldsymbol{A})=\psi(\boldsymbol{A}) \boldsymbol{P}_{2 n}^{+} \boldsymbol{P}_{2 n}$;

f) $\boldsymbol{Q}_{2 m}=\boldsymbol{Q}_{2 m}^{+}$.

2.3 Manifold modeling of vector sensors in the conformal array

In this subsection, we will combine the electromagnetic vector sensors with the conformal array, and present a unified model based on geometric algebra to estimate the DOAs. To illustrate the versatility of this algorithm, we consider a $M \times N$ cylindrical conformal array as shown in Fig. 3. The array contains $N$ uniformly spaced rings on the surface. In addition, there are $M$ electromagnetic vector sensors distributed on each ring. The angle between two consecutive elements on the same

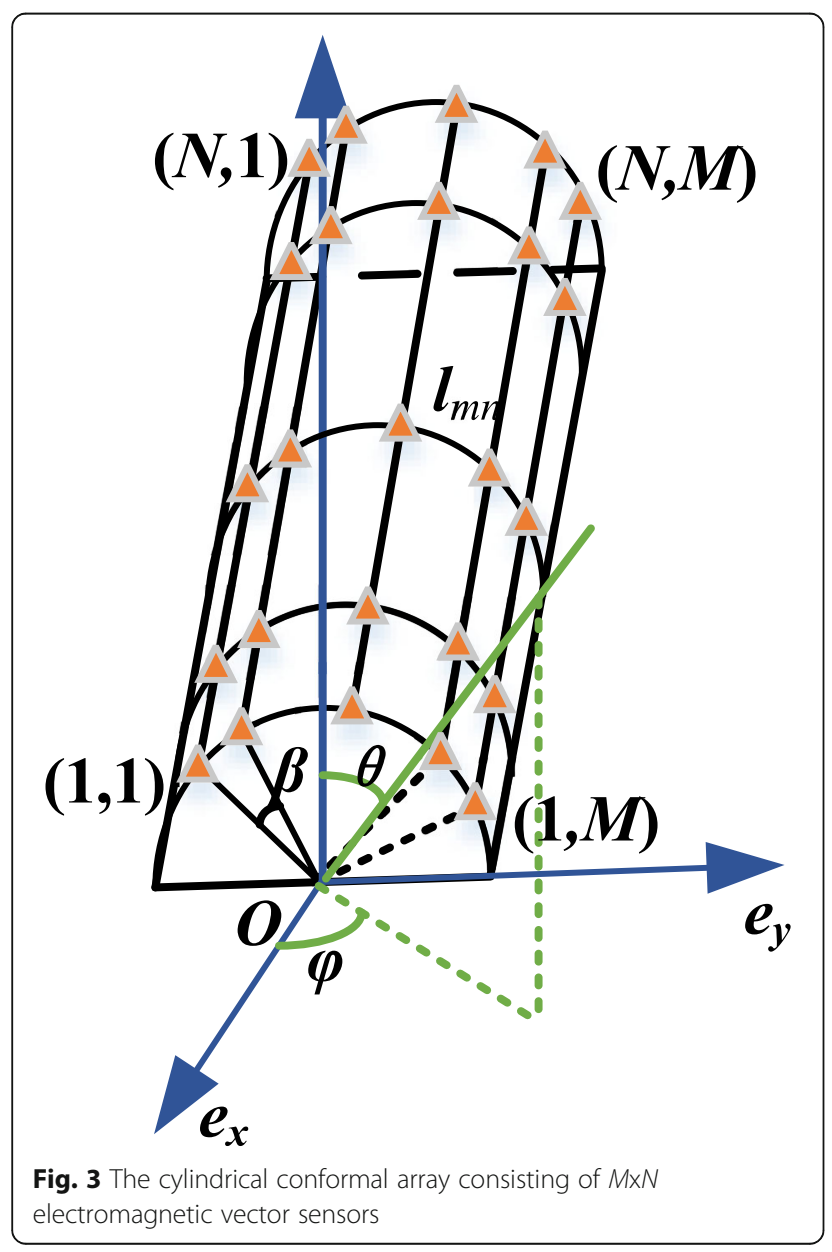

ring is $\beta$. In addition, the radius of the cylinder and the distance between adjacent rings are $R$ and $W$, respectively.

Since the electromagnetic vector sensor consists of six spatially collocated antennas, the three electric field components $\left(E_{x}, E_{y}, E_{z}\right)$ and the three magnetic field components $\left(H_{x} . H_{y}, H_{z}\right)$ can be measured simultaneously. Thus, we can use two multivectors, $X_{e}$ and $X_{h}$, to represent the electric field signal and the magnetic field signal, respectively.

$$
\begin{aligned}
& X_{e}=E_{x} \boldsymbol{e}_{x}+E_{y} \boldsymbol{e}_{y}+E_{z} \boldsymbol{e}_{z} \\
& X_{h}=H_{x} \boldsymbol{e}_{x}+H_{y} \boldsymbol{e}_{y}+H_{z} \boldsymbol{e}_{z}
\end{aligned}
$$

Similarly, the noise can be written as

$$
\begin{aligned}
& N_{e}=N_{E x} \boldsymbol{e}_{x}+N_{E y} \boldsymbol{e}_{y}+N_{E z} \boldsymbol{e}_{z} \\
& N_{h}=N_{H x} \boldsymbol{e}_{x}+N_{H y} \boldsymbol{e}_{y}+N_{H z} \boldsymbol{e}_{z}
\end{aligned}
$$

Then, the output of single element can be obtained in the frame of geometric algebra.

$$
Y=X_{e}+\boldsymbol{e}_{x y z} X_{h}+N_{e}+\boldsymbol{e}_{x y z} N_{h}
$$

From (22), we see that $\boldsymbol{e}_{x y z}$ not only provides a vital link between electric field components and magnetic field components, but also offers the possibility to handle the data model in geometric algebra. Due to the limited length, the relationship between the two fields will be derived in Appendix 2. In addition, from (18, 19, 20, and 21 ), we see that the orthogonality of the signal components is reserved. Compared with the conventional methods, such as the long vector algorithm [8], this orthogonality constraint implies stronger relationships between the signal components. The proof can be seen in Appendix 3. And it is also the most important advantage of the output model. Using the Maxwell equations in the formalism of geometric algebra, Eq. (22) can be written in another shape.

$$
Y=(1+\boldsymbol{u}) S_{E}+N_{e}+\boldsymbol{e}_{x y z} N_{h}
$$

where

$$
\boldsymbol{u}=\cos \phi \sin \theta \boldsymbol{e}_{x}+\sin \phi \sin \theta \boldsymbol{e}_{y}+\cos \theta \boldsymbol{e}_{z}
$$

with $\boldsymbol{u}$ representing the unit vector of the signal propagation and $S_{E}$ being the complex envelope of the electric field. In addition, the signal has an elevation angle $\theta$ and an azimuth angle $\phi$. The derivation of (23) is omitted here and the interested reader will find more material in [10].

Considering the polarization information, the aforementioned complex envelope, $S_{E}$, can be written as

$$
S_{E}=\Theta \boldsymbol{h} S
$$


where $\boldsymbol{h}$ is the signal polarization vector [16] and can be described by the auxiliary polarization angle $(\gamma)$ and the polarization phase difference $(\eta)$, that is, $\boldsymbol{h}=$ $\left[\begin{array}{ll}\cos \gamma & \sin \gamma e^{\boldsymbol{e}_{x y z}} \eta\end{array}\right]^{\mathrm{T}}$. And $S$ is the multivector symbolizing the complex envelope of the signal. Moreover, the parameter $\Theta$ denotes the steering vector of the angle field [17] and is independent of the space location:

$$
\Theta=\left[\begin{array}{cc}
-\sin \phi & \cos \theta \cos \phi \\
\cos \phi & \cos \theta \sin \phi \\
0 & -\sin \theta
\end{array}\right]
$$

Thus, the polarized version of (23) can be expressed as

$$
Y=\underbrace{(1+\boldsymbol{u}) \Theta \boldsymbol{h} S}_{X}+\underbrace{N_{e}+\boldsymbol{e}_{x y z} N_{h}}_{N_{y}}
$$

As stated above, the cylindrical conformal array is composed of $M \times N$ elements. In addition, suppose that there are $K$ narrowband sources impinging on the array. The manifold of the conformal array as shown in Fig. 3 corresponding to the $k$ th source is

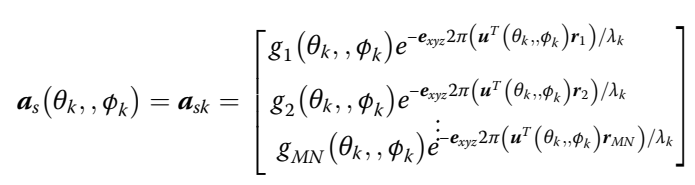

where $g_{m n}\left(\theta_{k}, \phi_{k}\right), m=1,2, \ldots, M, n=1,2, \ldots, N$ is the element pattern in the array global Cartesian coordinate system. In subsequent equations the range of $m$ and $n$ is the same and is omitted. $\boldsymbol{R}_{m n}$ and $\lambda_{k}$ are the $(m, n)$ th element location vector and the $k$ th signal wavelength respectively. The received signals of the array are the superposition of the response of each signal, the output can be expressed as

$$
\boldsymbol{Y}=\sum_{k=1}^{K} \boldsymbol{a}_{s k} X_{k}+\boldsymbol{N}_{\boldsymbol{y}}=\boldsymbol{A}\left(\theta_{k},, \phi_{k}, \gamma_{k}, \eta_{k}\right) \boldsymbol{S}+\boldsymbol{N}_{\boldsymbol{y}}
$$

where $X_{k}$ is a special case of $X$ regarding the $k$ th source. And

$$
\begin{aligned}
& \boldsymbol{S}=\left[\begin{array}{llll}
S_{1} & S_{2} & \cdots & S_{K}
\end{array}\right]^{\mathrm{T}} \\
& \boldsymbol{N}_{\boldsymbol{y}}=\left[\begin{array}{llll}
N_{y 1} & N_{y 2} \cdots & N_{y K}
\end{array}\right]^{\mathrm{T}}
\end{aligned}
$$

with $S_{k}$ being the complex envelope of the $k$ th signal. For notational convenience, we will simply write $\boldsymbol{A}$ instead of $\boldsymbol{A}\left(\theta_{k}, \phi_{k}, \gamma_{k}, \eta_{k}\right)$ whenever there is no possibility of confusion.

Let us refer back to Eq. (28). It is worthwhile to note that the aforementioned manifold of the conformal array, $\boldsymbol{a}_{s k}$, is derived under the global coordinate system. The azimuth and elevation angles are defined in Fig. 3. In most ready-made algorithms, the element pattern, $g_{m n}\left(\theta_{k}, \phi_{k}\right)$, is always considered to be identical. Nevertheless, due to the effects of the curvature of conformal carriers, the above assumption is not satisfied in the cylindrical conformal array.

In what follows, we will use the rotor in geometric algebra to model the conformal array, together with the vector-sensor array. The most important advantage of geometric algebra in analyzing conformal arrays is that we are able to express the geometry and the physics in a coordinate-free language. As stated above, the rotor can be used to realize the rotation between the two coordinate systems. Thus, we define the local coordinate system of the $(m, n)$ th element as shown in Fig. 4.

The $\boldsymbol{e}_{x m n}$ axis is the same as $\boldsymbol{e}_{x}$ axis in the global coordinate system, $\boldsymbol{e}_{z m n}$ is perpendicular to the element surface and $\boldsymbol{e}_{y m n}$ is tangent to the surface which can form a standard Cartesian coordinate system. Then, transforming the global coordinate into the local one is equivalent to rotating the global coordinate about $\boldsymbol{e}_{x}$ axis. The corresponding rotation angle is

$$
\xi=(m-1) \beta-\frac{M-1}{2} \beta=\left(m-\frac{M+1}{2}\right) \beta
$$

Substituting $\boldsymbol{e}_{z}$ and $\boldsymbol{e}_{y}$ for $\boldsymbol{b}$ and $\boldsymbol{a}$, respectively (see Appendix 1, the exponential form of the rotor), the rotor is

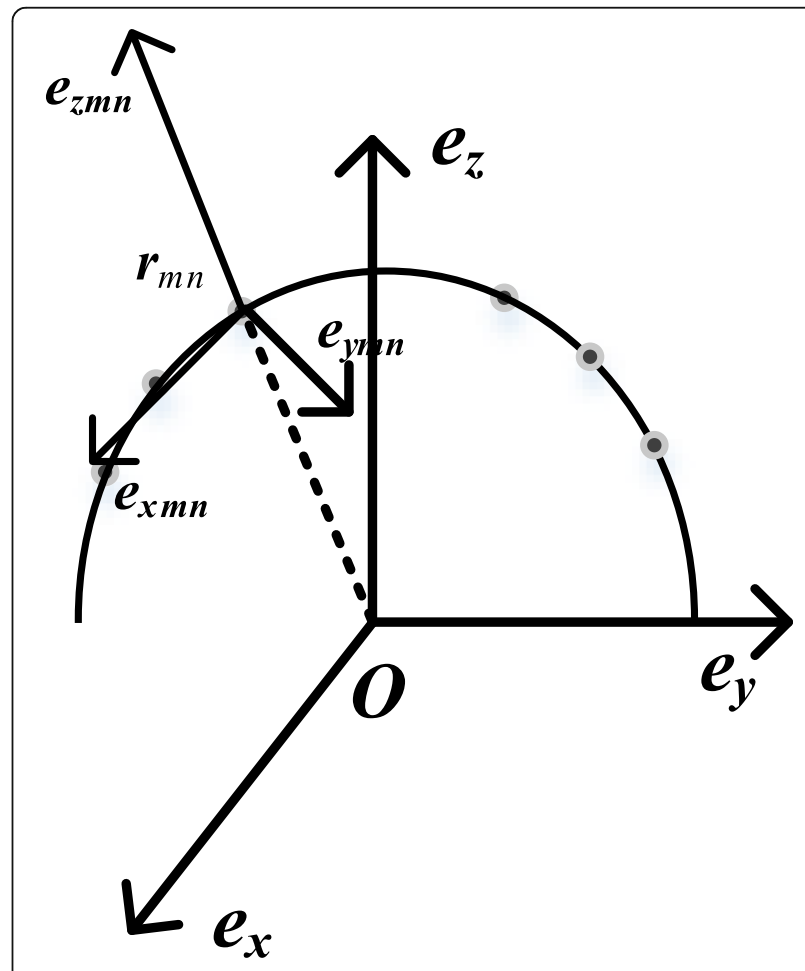

Fig. 4 The local coordinate of the $(m, n)$ th element 


$$
\boldsymbol{R}_{\boldsymbol{m} n}=e^{-\left(\boldsymbol{e}_{z} \wedge \boldsymbol{e}_{y}\right) \frac{\varepsilon}{2}}
$$

Utilizing $\boldsymbol{E}_{3}=\boldsymbol{e}_{x} \boldsymbol{e}_{y} \boldsymbol{e}_{z}$ as the pseudo-scalar in 3-D Euclidean space, Eq. (33) can be further simplified

$$
\boldsymbol{R}_{\boldsymbol{m} n}=e^{\boldsymbol{E}_{3} \boldsymbol{e}_{x \frac{\varepsilon}{2}}}
$$

Through (11), we can acquire the standard orthogonal basis in the local coordinate. And the specific procedure can refer to Appendix 4. We herein directly give the results

$$
\begin{aligned}
& \boldsymbol{e}_{x m n}=\boldsymbol{R}_{m n} \boldsymbol{e}_{x} \boldsymbol{R}_{m n^{-1}}=\boldsymbol{e}_{x} \\
& \boldsymbol{e}_{m n n}=\boldsymbol{R}_{m n} \boldsymbol{e}_{y} \boldsymbol{R}_{m n^{1}}=\cos \left[\left(m-\frac{M+1}{2}\right) \beta\right] \boldsymbol{e}_{y^{-}} \sin \left[\left(m-\frac{M+1}{2}\right) \beta\right] \boldsymbol{e}_{z} \\
& \boldsymbol{e}_{z m n}=\boldsymbol{R}_{m n} \boldsymbol{e}_{z} \boldsymbol{R}_{m n^{-1}}=\sin \left[\left(m-\frac{M+1}{2}\right) \beta\right] \boldsymbol{e}_{y}+\cos \left[\left(m-\frac{M+1}{2}\right) \beta\right] \boldsymbol{e}_{z}
\end{aligned}
$$

Thus, from (35, 36 and 37), we can obtain the element pattern, $g_{m n}\left(\theta_{k}, \phi_{k}\right)$.

Up to present, the remaining unknown variable is the location vector. From Fig. 3, we can obtain its specific expression

$$
\boldsymbol{r}_{m n}=(-n \delta) \boldsymbol{e}_{x}+\left(R \sin \left(m-\frac{M+1}{2}\right) \beta\right) \boldsymbol{e}_{y}+\left(R \cos \left(m-\frac{M+1}{2}\right) \beta\right) \boldsymbol{e}_{z}
$$

where $\delta$ means the spacing between adjacent rings. Then, the mainfold of the conformal vector-sensor array, $A$, can be obtained.

Since the geometric algebra is introduced in modeling the manifold, the eigendecomposition is different from the conventional methods, such as [18]. In fact, similar to the quaternion case [19], the noncommutativity of the geometric product leads to two possible eigenvalues, namely the left and the right eigenvalue. However, in this paper, we select the right eigenvalue since the right eigendecomposition of $\boldsymbol{A}$ can be converted to the right eigendecomposition of the CRM.

We construct the covariance matrix

$$
\boldsymbol{R}_{\boldsymbol{Y}}=E\left\{\boldsymbol{Y} \boldsymbol{Y}^{+}\right\}=\boldsymbol{A} E\left\{\boldsymbol{S \boldsymbol { S } ^ { + }}\right\} \boldsymbol{A}^{+}+6 \sigma^{2} \boldsymbol{I}_{M N}
$$

Here, we assume that the noise is identical and uncorrelated from element to element, with covariance $\sigma^{2}$.

For $\boldsymbol{R}_{\boldsymbol{Y}}$ is a unitary matrix, its eigendecomposition can be written as

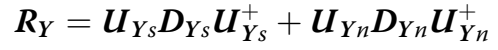

Where $\boldsymbol{U}_{Y S}$ is the $M N x K$ matrix composed of the $K$ eigenvectors corresponding to the $K$ largest eigenvalues of $\boldsymbol{R}_{\boldsymbol{Y}}$, termed as the signal subspace. $\boldsymbol{U}_{\boldsymbol{Y}}$ represents the matrix composed of the eigenvectors corresponding to the $2 M-K$ smaller eigenvalues, i.e., the noise subspace. According to the principles of the MUSIC algorithm, the array manifold spans the signal subspace and is orthogonal to the noise subspace. In this case, we have

$$
\boldsymbol{A}^{+} \boldsymbol{U}_{\boldsymbol{Y} n}=\mathbf{0}_{2 M-K}
$$

where $\mathbf{0}_{2 M-K}$ is a $2 M-K$ row vector with all elements equal zero. The proof can be seen in Appendix 5.

In practice, $\boldsymbol{R}_{\boldsymbol{Y}}=\frac{1}{L} \sum_{l=1}^{L} \boldsymbol{Y} \boldsymbol{Y}^{+}=\boldsymbol{U}_{\boldsymbol{Y}_{s}} \boldsymbol{D}_{Y_{s}} \boldsymbol{U}_{\boldsymbol{Y}}^{+}+\boldsymbol{U}_{\boldsymbol{Y} n} \boldsymbol{D}_{\boldsymbol{Y} n}$ $\boldsymbol{U}_{\boldsymbol{Y} n}^{+}$, the maximum likelihood estimation of $\boldsymbol{R}_{\boldsymbol{Y}}$, is always used as the covariance matrix. Among which, $L$ represents the number of snapshots. In this case, (41) becomes

$$
\boldsymbol{A}^{+} \boldsymbol{U}_{\boldsymbol{Y} n} \approx \mathbf{0}_{2 M-K}
$$

Up to present, the DOA estimation model of conformal vector-sensor array has been established. This is also the focus of our paper. The contents of constructing the spatial spectra and searching the peak are omitted here. The readers can refer to literature [18]. It is worthwhile to note that in introducing the rotor, the spatial location of the sensor is not required. Then, the proposed method can be easily extended to other arrays.

It is also worthwhile to note that $\left\{\boldsymbol{e}_{x}, \boldsymbol{e}_{y} \boldsymbol{e}_{z}\right\}$ is not only the basis for the multivector in the vector-sensor array, but also represents the coordinate in the conformal array. And, it can be used for transformation between the global and the local coordinates with the help of the rotor. Under this circumstance, there are some links between those two arrays. The commonality is one of the motivations for establishing a unified model to estimate the DOAs.

\section{Complexity analysis}

To better explain the superiority of the geometric algebra in modeling the conformal vector-sensor array, we will introduce the computational complexity from the standpoint of deriving the manifold. And, the computational burden is evaluated in terms of the number of multiplications, additions, and transpositions.

To this end, we will briefly introduce the conventional methods of analysis based on Euler angle in this section. Generally, the transformations between the element local coordinates and the array global coordinates may be implemented by three continuous Euler rotations [3]. The specific rotation matrix can be expressed as

$$
\begin{aligned}
\boldsymbol{R}(C, D, F) & =\boldsymbol{R}_{x}(C) \boldsymbol{R}_{y}(D) \boldsymbol{R}_{z}(F) \\
& =\left[\begin{array}{ccc}
1 & 0 & 0 \\
0 & \cos C & -\sin C \\
0 & \sin C & \cos C
\end{array}\right]\left[\begin{array}{ccc}
\cos D & 0 & -\sin D \\
0 & 1 & 0 \\
\sin D & 0 & \cos D
\end{array}\right]\left[\begin{array}{ccc}
\cos F & -\sin F & 0 \\
\sin F & \cos F & 0 \\
0 & 0 & 1
\end{array}\right] \\
& =\left[\begin{array}{ccc}
\cos D \cos F & -\cos D \sin F & -\sin D \\
\cos C \sin F-\cos F \sin C \sin D & \cos C \cos F+\sin C \sin D \sin F & -\cos D \sin C \\
\sin C \sin F+\cos C \cos F \sin D & \cos F \sin C-\cos C \sin D \sin F & \cos C \cos D
\end{array}\right]
\end{aligned}
$$

where $C, D$, and $F$ are, respectively, three consecutive 
Euler rotation angles about $\boldsymbol{e}_{x}$ axis, $\boldsymbol{e}_{y}$ axis and $\boldsymbol{e}_{z}$ axis. The matrices $\boldsymbol{R}_{x}(C), \boldsymbol{R}_{y}(D)$, and $\boldsymbol{R}_{z}(F)$ are the corresponding Euler rotation matrices. It is noted that two successive Euler rotations are usually adequate to deal with the cylindrical conformal array. The third Euler rotation matrix is added here to cope with some irregular or special conformal arrays. Additionally, we know the rotation matrix is invertible from Eq. (43). Consequently, taking the inversion with respect to $\boldsymbol{R}(C, D, F)$, we have

$$
\begin{aligned}
\boldsymbol{R}(C, D, F)^{-1} & =\boldsymbol{R}_{z^{-1}}(F) \boldsymbol{R}_{y^{-1}}(D) \boldsymbol{R}_{x^{-1}}(C) \\
& =\left[\begin{array}{ccc}
\cos F & \sin F & 0 \\
-\sin F & \cos F & 0 \\
0 & 0 & 1
\end{array}\right]\left[\begin{array}{ccc}
\cos D & 0 & \sin D \\
0 & 1 & 0 \\
-\sin D & 0 & \cos D
\end{array}\right]\left[\begin{array}{ccc}
1 & 0 & 0 \\
0 & \cos C & \sin C \\
0 & -\sin C & \cos C
\end{array}\right] \\
& =\left[\begin{array}{ccc}
\cos D \cos F & \cos C \sin F-\cos F \sin C \sin D & \sin C \sin F+\cos C \cos F \sin D \\
-\cos D \sin F & \cos C \cos F+\sin C \sin D \sin F & \cos F \sin C-\cos C \sin D \sin F \\
-\sin D & -\cos D \sin C & \cos C \cos D
\end{array}\right]
\end{aligned}
$$

Combining Eq. (43) with Eq. (44), it is not hard to find that

$$
\boldsymbol{R}(C, D, F)^{-1}=\boldsymbol{R}(C, D, F)^{\mathrm{T}}
$$

Thus, $\boldsymbol{R}(C, D, F)$ is the so-called orthogonal matrix. In this case, transforming the local coordinate into the global one is equivalent to imposing the transposition/inversion with respect to the above rotation matrix. If we model the conformal array based on the Euler angle, three matrix multiplications and one matrix transposition are required for each element.

In fact, the matrix operations are essentially the multiplications and the additions between elements. To quantify this, the amounts of multiplications and additions of the two methods (i.e., the proposed method and the Euler angle method) are calculated, respectively. The corresponding results are shown in Table 2 . We assume that one matrix transposition is considered as one multiplication or addition operation. And it is obvious that the multiplication between two $3 \times 3$ matrices involves $9 \times 3$ multiplications and $9 \times 2$ additions. For convenience, the multiplication and the addition are collectively referred to as the operation. Then, Eq. (43) contains $2 \times 9 \times 3+2 \times 9 \times 2$ operations. For the conformal array consisting of $M \times N$ electromagnetic vector sensors, the transformation between different coordinates involves $91 \times 6 \times M N$ operations. Compared with the Euler rotation angle, the proposed method effectively avoids the cumbersome matrix transformations. From Eqs. (35, 36, and 37), we know $\boldsymbol{e}_{y m n}$ and $\boldsymbol{e}_{z m n}$ are independent of $\boldsymbol{e}_{x}$. In addition, $\boldsymbol{e}_{x m n}$ can be obtained directly from Eq. (35) without extra operations. Thus, Eqs. (35, 36 and 37), can be expressed as a $2 \times 2$ matrix. While using the rotor to establish the array manifold, the computational process is equivalent to a $2 \times 2$ matrix multiplied by a $2 \times 1$ vector. In this case, the operations for each element involve four multiplications and two additions. The total amount of operations is $6 \times 6 \times M N$. Thus, the geometric algebra-based method significantly decreases the computational burden.

In general, the Euler rotation and its matrix representation cannot intuitively exhibit the complete procedure. In addition, as the configuration of the conformal array becomes more irregular and complex, the level of complexity involved in the transformations and the number of calculations required increases largely.

\section{Simulation results}

In this section, Monte-Carlo simulation experiments are used to verify the effectiveness of the proposed algorithm. The array structure is shown in Fig. 3. Among which, we select $M$ and $N$ as 4 and 4 , respectively. The angle between two consecutive elements on the same ring, $\beta$, is $5^{\circ}$. The number of snapshots, $L$, is 200 . Under these premises, 200 independent simulation experiments are carried out. The root mean square error (RMSE) is utilized as the performance measure and is defined as

$$
R M S E=\sqrt{\frac{1}{200} \sum_{i=1}^{200}\left[\left(\hat{\theta}_{i}-\theta_{i}\right)^{2}+\left(\hat{\phi}_{i}-\phi_{i}\right)^{2}\right]}
$$

where $\left\{\theta_{i}, \phi_{i}\right\}$ are the estimates of elevation angles and azimuth angles, respectively, at the $i$ th run.

Provided that there are three polarized signals can be received. The incident angles are $\left(10^{\circ}, 15^{\circ}\right),\left(35^{\circ}, 40^{\circ}\right)$, and $\left(60^{\circ}, 35^{\circ}\right)$, respectively. The corresponding polarization auxiliary angles and the polarization phase differences are $\left(15^{\circ}, 25^{\circ}\right),\left(30^{\circ}, 45^{\circ}\right)$, and $\left(50^{\circ}, 65^{\circ}\right)$. Figure 5 shows the simulation results of the proposed algorithm. The position of the spectrum peak represents the possible DOA. Intuitively, the estimation accuracy of the proposed algorithm is high.

To better demonstrate the performance of the proposed method, Qi's method [3] and Gao's algorithm [20] are included for comparison. We study the performance with a varying SNR from 0 to $30 \mathrm{~dB}$. Without loss of generality, we select the first source (T1) and the second source (T2), respectively, to verify it. Figure 6 shows the RMSE versus SNR with the snapshots being 200. It can be seen that the proposed method outperforms the Qi's method [3] by preserving the orthogonality of the received signal components. In addition, the performance of Gao's algorithm is also worse than the proposed one. Two main reasons lead to this difference. Firstly, the proposed method imposes stronger constraints between the components of the signals. Secondly, the conformal array in [20] essentially belongs to the scalar array from the standpoint of elements while the conformal vectorsensor array presented in this paper belongs to the vector array. And the vector array contains more signal 
Table 2 The computational complexity of the proposed method and Euler angle

\begin{tabular}{lllll}
\hline & Multiplications & Additions & Transpositions & Operations \\
\hline Euler angle & $2 \times 9 \times 3 \times 6 \times M N$ & $2 \times 9 \times 2 \times 6 \times M N$ & $6 \times M N$ & $91 \times 6 \times M N$ \\
Proposed method & $4 \times 6 \times M N$ & $2 \times 6 \times M N$ & 0 & $6 \times 6 \times M N$
\end{tabular}

information compared with the scalar array. Moreover, in contrast to those two algorithms, the proposed one effectively avoids the cumbersome matrix transformations, and therefore, has a much lower computational complexity. It is noted that, for the statistical data have certain randomness, the simulation curve in Fig. 6 is not smooth.

Figure 7 illustrates the RMSE versus the number of snapshots with the SNR fixed at $10 \mathrm{~dB}$. Compared with Fig. 6, we can draw similar conclusions. In particular, if we pick the points with snapshots being 300 and 600, respectively, we may find that the corresponding RMSEs are 0.5805 and 0.2902 . This means the former value is nearly twice as much as the latter one. In fact, these improvements can be predicted from the derivation of CRB. The specific derivation process can refer to literature [21]. The number of snapshots can be extracted from the Fisher information matrix. Moreover, the CRB is found as the element of the inverse of that matrix. So, we can conclude that the RMSE is inversely proportional to $L$.

To better demonstrate the computational efficiency, the specific operations, such as the multiplications, additions, and transpositions, are simulated in Fig. 8. The value of the $x$-axis (or the abscissa) represents the product of $M$ and $N$. It can be seen that the multiplications take up the most resources. Compared with Euler rotation angles, the proposed method reduces the computation by one order of magnitude. Thus, the proposed algorithm provides the possibility for real-time processing.

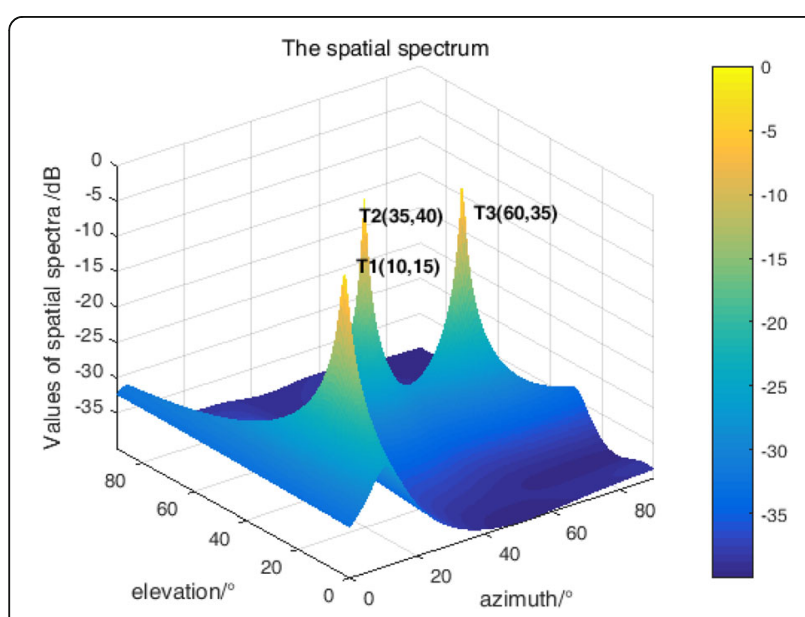

Fig. 5 The spatial spectrum of the proposed algorithm

\section{Conclusions}

In this correspondence, we combine the electromagnetic vector sensors with the conformal array, and present a unified model based on geometric algebra to estimate the DOAs. Compared with existing methods, the proposed one has two main advantages. Firstly, it can give a more accurate estimation by preserving the orthogonality of the signal components. Secondly, it avoids the cumbersome matrix operations while performing the coordinate transformations, and therefore, has a much lower computational complexity. In addition, it has a strong commonality, that is to say, it is not limited to any specific conformal array. The simulation results verify the effectiveness of the proposed method.

\section{Appendix 1}

6.1 Here we will give a brief proof to demonstrate that the rotation must be over twice the angle between $a$ and $b$

To proceed further, we rewrite $\boldsymbol{R}$ according to the definition of the geometric product:

$$
\boldsymbol{R}=\boldsymbol{b a}=\boldsymbol{b} \cdot \boldsymbol{a}+\boldsymbol{b} \wedge \boldsymbol{a}
$$

Here, we consider the case that the vectors are unit length. This assumption is reasonable, because the basic vectors of the Cartesian coordinate system satisfy it as well. The geometric product of $\boldsymbol{b} \wedge \boldsymbol{a}$ itself is:

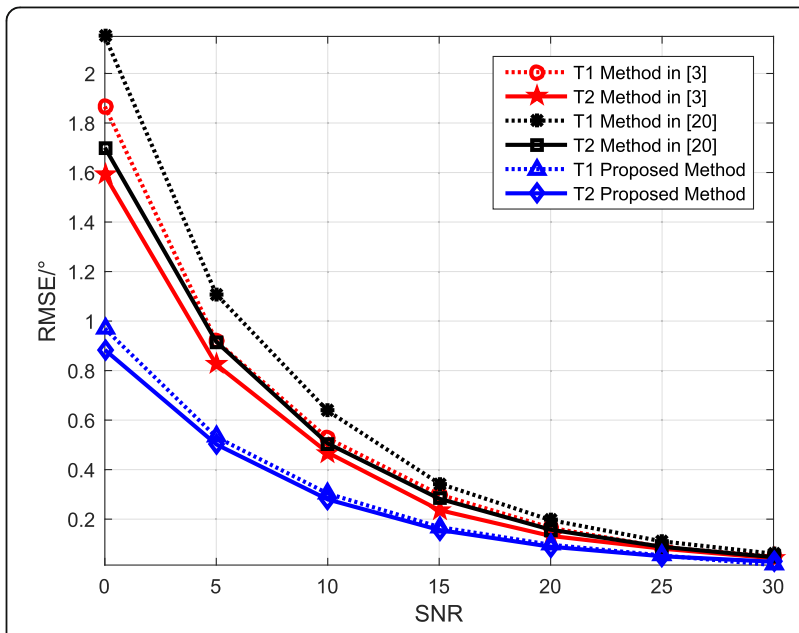

Fig. 6 RMSE versus SNR with the snapshots being 200 


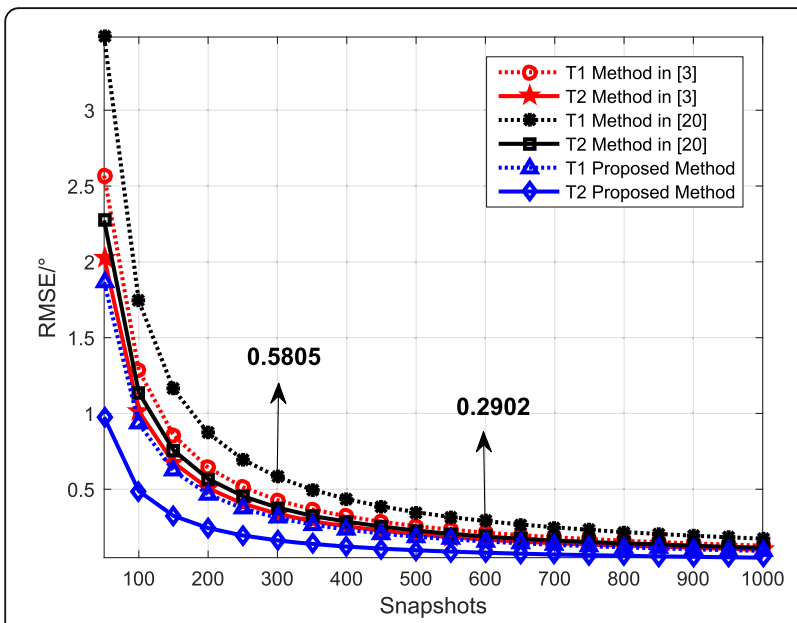

Fig. 7 RMSE versus snapshots with the SNR fixed at $10 \mathrm{~dB}$

$$
\begin{aligned}
(\boldsymbol{b} \wedge \boldsymbol{a})(\boldsymbol{b} \wedge \boldsymbol{a}) & =(\boldsymbol{b} \boldsymbol{a}-\boldsymbol{b} \cdot \boldsymbol{a})(\boldsymbol{b} \cdot \boldsymbol{a}-\boldsymbol{a} \boldsymbol{b}) \\
& =\boldsymbol{b} \cdot \boldsymbol{a}(\boldsymbol{b} \boldsymbol{a}+\boldsymbol{a} \boldsymbol{b})-(\boldsymbol{b} \cdot \boldsymbol{a})^{2}-\boldsymbol{b} \boldsymbol{a} \boldsymbol{a} \boldsymbol{b} \\
& =\boldsymbol{b} \cdot \boldsymbol{a}(2 \boldsymbol{b} \cdot \boldsymbol{a})-(\boldsymbol{b} \cdot \boldsymbol{a})^{2}-\boldsymbol{b}(\boldsymbol{a} \boldsymbol{a}) \boldsymbol{b} \\
& =(\boldsymbol{b} \cdot \boldsymbol{a})^{2}-\boldsymbol{b} \boldsymbol{b} \\
& =\cos ^{2} \theta-1 \\
& =-\sin ^{2} \theta
\end{aligned}
$$

Thus, we define the 2-blade $\boldsymbol{E}_{2}$ :

$$
\boldsymbol{E}_{2}=\frac{\boldsymbol{b} \wedge \boldsymbol{a}}{\sin \theta}
$$

$\boldsymbol{R}$ can be further simplified by substituting (49) into (47):

$$
\boldsymbol{R}=\cos \theta-\boldsymbol{E}_{2} \sin \theta
$$

The expression is similar to the polar decomposition of a complex number with the unit imaginary replaced by the 2-blade $\boldsymbol{E}_{2}$. (50) can also be written as the exponentials of $\boldsymbol{E}_{2}$ :

$$
\boldsymbol{R}=e^{-E_{2} \theta}
$$

This formalism is more useful for the log-space of rotors is linear. We split $\boldsymbol{x}$ into a part $\left(\boldsymbol{x}_{p}\right)$ parallel to $\boldsymbol{b} \Lambda$ $\boldsymbol{a}$-plane and a part $\left(\boldsymbol{x}_{o}\right)$ orthogonal to $\boldsymbol{b} \wedge \boldsymbol{a}$-plane. Then, $\boldsymbol{x}_{o}$ is not affected by the application $\boldsymbol{R}$. And we infer that the rotation must be in the $\boldsymbol{b} \wedge \boldsymbol{a}$-plane. As stated above, the rotation consists of two successive reflections which are orthogonal (angle-preserving) transformations. Thus, it allows us to pick any vector in the $\boldsymbol{b} \wedge \boldsymbol{a}$-plane to determine the angle. Without loss of generality, we choose vector $\boldsymbol{a}$, and construct the "sandwich product" $\boldsymbol{R a R}^{-1}$ as shown in (11):

$$
\operatorname{RaR}^{-1}=\mathrm{baaa}^{-1} \boldsymbol{b}^{-1}=\boldsymbol{b a b}^{-1}
$$

where $\boldsymbol{b} \boldsymbol{a} \boldsymbol{b}^{-1}$ is the reflection of $\boldsymbol{a}$ in $\boldsymbol{b}$. From this it is clear that the rotation must be over twice the angle between $\boldsymbol{a}$ and $\boldsymbol{b}$, since the angle between $\boldsymbol{a}$ and $\boldsymbol{b} \boldsymbol{a} \boldsymbol{b}^{-1}$ is twice the angle between $\boldsymbol{a}$ and $\boldsymbol{b}$. The negative signature in (51) represents the rotation direction.

\section{Appendix 2}

7.1 We demonstrate how $e_{x y z}$ links the electric field with the magnetic field

First, let us refer back to the famous Maxwell equations described by the vector algebra are

$$
\begin{aligned}
& \nabla \times \boldsymbol{E}=-\mu \frac{\partial \boldsymbol{H}}{\partial t} \\
& \nabla \cdot \boldsymbol{E}=\frac{\rho}{\varepsilon} \\
& \nabla \times \boldsymbol{H}=\varepsilon \frac{\partial \boldsymbol{E}}{\partial t} \\
& \nabla \cdot \boldsymbol{H}=0
\end{aligned}
$$

where $\boldsymbol{E}=E_{x} \boldsymbol{e}_{x}+E_{y} \boldsymbol{e}_{y}+E_{z} \boldsymbol{e}_{z}$ and $\boldsymbol{H}=H_{x} \boldsymbol{e}_{x}+H_{y} \boldsymbol{e}_{y}+H_{z} \boldsymbol{e}_{z}$ are, respectively, the electric and magnetic fields. In addition, the parameters $\varepsilon, \mu$, and $\rho$ symbolize the

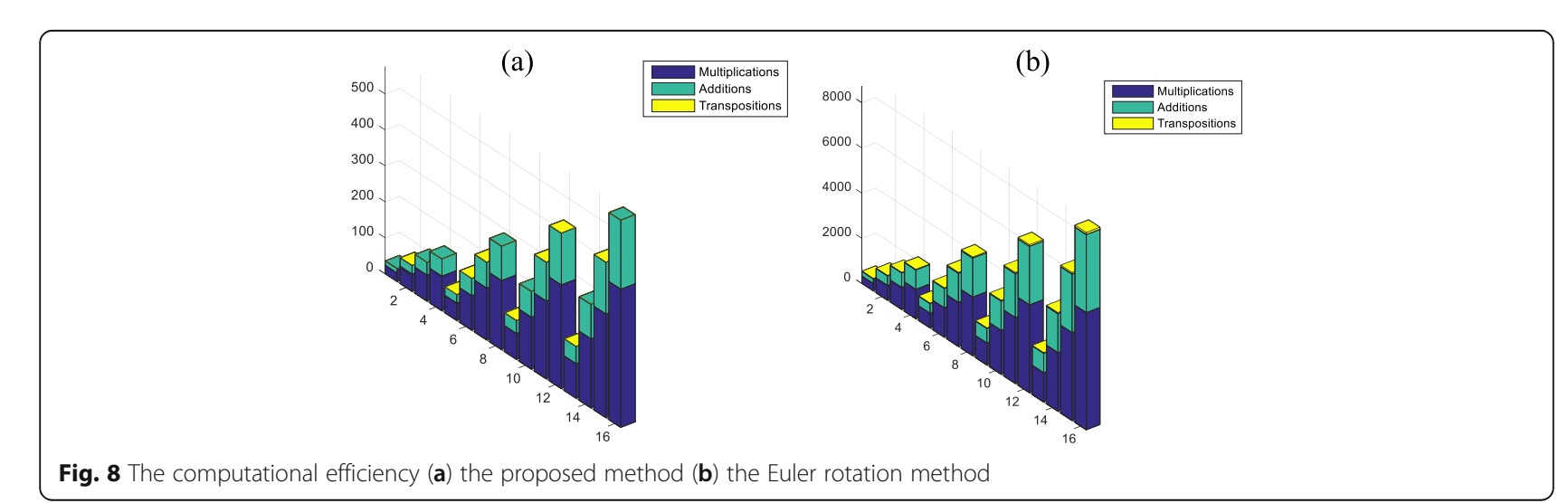


permittivity, the permeability, and the density of the source charges, respectively.

It is worthwhile to give some results before proceeding to the physical problems of interest:

$$
\begin{aligned}
& \boldsymbol{x} \wedge \boldsymbol{y}=\boldsymbol{e}_{x y z} \boldsymbol{x} \times \boldsymbol{y} \\
& \boldsymbol{x} \times \boldsymbol{y}=-\boldsymbol{x} \cdot\left(\boldsymbol{y} \boldsymbol{e}_{x y z}\right) \\
& \boldsymbol{x} \cdot \boldsymbol{y}=-\boldsymbol{e}_{x y z}\left(\boldsymbol{x} \wedge\left(\boldsymbol{e}_{x y z} \boldsymbol{y}\right)\right)
\end{aligned}
$$

Using Eqs. (57, 58 and 59), the Maxwell equations in geometric algebra can be expressed as follows:

$$
\begin{aligned}
& \nabla \wedge \boldsymbol{E}+\mu \frac{\partial \boldsymbol{H}}{\partial t} \boldsymbol{e}_{x y z}=0 \\
& \nabla \cdot \boldsymbol{E}=\frac{\rho}{\varepsilon} \\
& \nabla \cdot\left(\boldsymbol{H} \boldsymbol{e}_{x y z}\right)+\varepsilon \frac{\partial \boldsymbol{E}}{\partial t}=0 \\
& \nabla \wedge\left(\boldsymbol{H e}_{x y z}\right)=0
\end{aligned}
$$

Since the arriving signals are assumed to be far-field, so the signals received on different positions differ only by a transmission delay, that is,

$$
\begin{aligned}
& \boldsymbol{E}(\boldsymbol{r}, t)=\boldsymbol{E}(0, t-\tau) \\
& \boldsymbol{H}(\boldsymbol{r}, t)=\boldsymbol{H}(0, t-\tau)
\end{aligned}
$$

where $\boldsymbol{r}=\boldsymbol{r}_{x} \boldsymbol{e}_{x}+\boldsymbol{r}_{y} \boldsymbol{e}_{y}+\boldsymbol{r}_{z} \boldsymbol{e}_{z}$, and $\tau$ is the so-called transmission delay,

$$
\tau=\frac{\boldsymbol{u} \cdot \boldsymbol{r}}{c}
$$

with $c$ representing the propagation speed and $\boldsymbol{u}$ denoting the signal propagation as defined in (24). Let

$$
\begin{aligned}
& \boldsymbol{E}(t)=\boldsymbol{E}(0, t)=\boldsymbol{E}_{x}(t) \boldsymbol{e}_{x}+\boldsymbol{E}_{y}(t) \boldsymbol{e}_{y}+\boldsymbol{E}_{z}(t) \boldsymbol{e}_{z} \\
& \boldsymbol{H}(t)=\boldsymbol{H}(0, t)=\boldsymbol{H}_{x}(t) \boldsymbol{e}_{x}+\boldsymbol{H}_{y}(t) \boldsymbol{e}_{y}+\boldsymbol{H}_{z}(t) \boldsymbol{e}_{z}
\end{aligned}
$$

Then,

$$
\begin{aligned}
& \boldsymbol{E}(\boldsymbol{r}, t)=\boldsymbol{E}(t-\tau) \\
& \boldsymbol{H}(\boldsymbol{r}, t)=\boldsymbol{H}(t-\tau)
\end{aligned}
$$

For plane waves, we know

$$
\nabla=\frac{u}{c} \frac{\partial}{\partial t}
$$

Substituting Eqs. (67, 68, 69, 70 and 71) into Maxwell equations, we have

$$
\begin{aligned}
& \frac{\boldsymbol{u}}{c} \wedge \dot{\boldsymbol{E}}(t)+\mu \dot{\boldsymbol{H}}(t) \boldsymbol{e}_{x y z}=0 \\
& \frac{\boldsymbol{u}}{c} \cdot \dot{\boldsymbol{E}}(t)=\frac{\rho}{\varepsilon} \\
& \frac{\boldsymbol{u}}{c} \cdot\left(\dot{\boldsymbol{H}}(t) \boldsymbol{e}_{x y z}\right)+\varepsilon \dot{\boldsymbol{E}}(t)=0 \\
& \frac{\boldsymbol{u}}{c} \wedge\left(\dot{\boldsymbol{H}}(t) \boldsymbol{e}_{x y z}\right)=0
\end{aligned}
$$

where $\boldsymbol{E}(t)=\frac{d \boldsymbol{E}}{d t}$ and $\boldsymbol{H}(t)=\frac{d \boldsymbol{H}}{d t}$.

From (75), we know $\boldsymbol{u}$ is in the plane $\boldsymbol{H}(t) \boldsymbol{e}_{x y z}$. In addition, combining the geometric implication of the inner product with Eq. (74), we know $\boldsymbol{E}(t)$ is the orthogonal complement of $\boldsymbol{u}$ in the plane $\boldsymbol{H}(t) \boldsymbol{e}_{x y z}$. That is to say, $\boldsymbol{E}(t)$ and $\boldsymbol{u}$ are orthogonal in that plane. Thus, the parameter $\rho$ in (73) equals to zero. Then, Eqs. $(72,73)$ are equivalent to Eqs. $(74,75)$. We rewrite $(73)$,

$$
\frac{\boldsymbol{u}}{c} \cdot \dot{\boldsymbol{E}}(t)=0
$$

Combining (72) and (76), we have

$$
\sqrt{\frac{\mu}{\varepsilon}} \dot{H}(t) \boldsymbol{e}_{x y z}=\boldsymbol{u} \dot{\boldsymbol{E}}(t)
$$

where $\sqrt{\frac{\mu}{\varepsilon}}$ denotes the intrinsic impedance of the medium.

Integrating (77) with respect to time $t$,

$$
\sqrt{\frac{\mu}{\varepsilon}} \dot{H}(t) \boldsymbol{e}_{x y z}=\boldsymbol{u} \dot{\boldsymbol{E}}(t)+q
$$

where $q$ is a constant and equals to zero in the far-field assumption.

Up to this point, we have derived how $\boldsymbol{e}_{x y z}$ links the electric field with the magnetic field.

\section{Appendix 3}

8.1 We show the orthogonality constraint implies stronger relationships between the signal components

Consider two electric field multivectors $X_{e 1}, X_{e 2}$, with their expressions given by

$$
\begin{aligned}
& X_{e 1}=E_{x 1} \boldsymbol{e}_{x}+E_{y 1} \boldsymbol{e}_{y}+E_{z 1} \boldsymbol{e}_{z} \\
& X_{e 2}=E_{x 2} \boldsymbol{e}_{x}+E_{y 2} \boldsymbol{e}_{y}+E_{z 2} \boldsymbol{e}_{z}
\end{aligned}
$$

By imposing the orthogonality for the two multivectors

$$
\left\langle X_{e 2}, \quad X_{e 1}\right\rangle_{g}=X_{e 1}^{\mathrm{H}} \cdot X_{e 2}=0
$$

where $\langle\cdot\rangle_{g}$ represents the inner product in geometric algebra.

We can get the following relationships between the signal components: 


$$
\begin{aligned}
& E_{x 1}^{\mathrm{H}} E_{x 2}+E_{y 1}^{\mathrm{H}} E_{y 2}+E_{z 1}^{\mathrm{H}} E_{z 2}=0 \\
& E_{x 2} E_{y 1}^{\mathrm{H}}=E_{y 2} E_{x 1}^{\mathrm{H}} \\
& E_{y 2} E_{z 1}^{\mathrm{H}}=E_{z 2} E_{y 1}^{\mathrm{H}} \\
& E_{x 2} E_{z 1}^{\mathrm{H}}=E_{z 2} E_{x 1}^{\mathrm{H}}
\end{aligned}
$$

However, for long vector algorithm, the multivectors are replaced by the vectors $\boldsymbol{x}_{e 1}, \boldsymbol{x}_{e 2}$, and correspondingly,

$$
\boldsymbol{x}_{e 1}=\left[\begin{array}{c}
E_{x 1} \\
E_{y 1} \\
E_{z 1}
\end{array}\right], \quad \boldsymbol{x}_{e 2}=\left[\begin{array}{c}
E_{x 2} \\
E_{y 2} \\
E_{z 2}
\end{array}\right]
$$

Similarly, imposing the orthogonality for the two vectors

$$
\left\langle x_{e 2}, \quad x_{e 1}\right\rangle_{v}=x_{e 1}^{\mathrm{H}} \cdot x_{e 2}=0
$$

where $\langle\cdot\rangle_{\nu}$ denotes the inner product between two vectors.

We can get the same result as in (82). However, Eqs. (83, 84 and 85) cannot be obtained. In other words, using geometric algebra to model the output imposes stronger constraints between the components of the vector sensor array.

\section{Appendix 4}

9.1 The detailed calculation procedures of Eqs. (35, 36 and 37), are given as follows. Since the derivations of Eqs. (36, 37) are similar to that of Eq. (35), we will take Eq. (35) as an example. And the other two equations can be obtained similarly. The derivation of (35) is

$$
\begin{aligned}
\boldsymbol{e}_{x m n} & =\boldsymbol{R}_{m n} \boldsymbol{e}_{x} \boldsymbol{R}_{m n^{-1}} \\
& =e^{\boldsymbol{E}_{3} \boldsymbol{e}_{x} \frac{\xi}{2}} \boldsymbol{e}_{x} \boldsymbol{E}_{3} \boldsymbol{e}_{x} \frac{\xi}{2} \\
& =\left(\cos \frac{\xi}{2}+\boldsymbol{E}_{3} \boldsymbol{e}_{x} \sin \frac{\xi}{2}\right) \boldsymbol{e}_{x}\left(\cos \frac{\xi}{2}-\boldsymbol{E}_{3} \boldsymbol{e}_{x} \sin \frac{\xi}{2}\right) \\
& =\left(\boldsymbol{e}_{x} \cos \frac{\xi}{2}+\boldsymbol{E}_{3} \sin \frac{\xi}{2}\right)\left(\cos \frac{\xi}{2}-\boldsymbol{E}_{3} \boldsymbol{e}_{x} \sin \frac{\xi}{2}\right) \\
& =\boldsymbol{e}_{x} \cos ^{2} \frac{\xi}{2}-\boldsymbol{e}_{x} \boldsymbol{E}_{3} \boldsymbol{e}_{x} \cos \frac{\xi}{2} \sin \frac{\xi}{2}+\boldsymbol{E}_{3} \sin \frac{\xi}{2} \cos \frac{\xi}{2}-\boldsymbol{E}_{3} \boldsymbol{E}_{3} \boldsymbol{e}_{x} \sin ^{2} \frac{\xi}{2} \\
= & \boldsymbol{e}_{x} \cos ^{2} \frac{\xi}{2}-\boldsymbol{e}_{x} \boldsymbol{e}_{x} \boldsymbol{e}_{y} \boldsymbol{e}_{z} \boldsymbol{e}_{x} \cos \frac{\xi}{2} \sin \frac{\xi}{2}+\boldsymbol{e}_{x} \boldsymbol{e}_{y} \boldsymbol{e}_{z} \sin \frac{\xi}{2} \cos \frac{\xi}{2}-\boldsymbol{e}_{x} \boldsymbol{e}_{y} \boldsymbol{e}_{z} \boldsymbol{e}_{x} \boldsymbol{e}_{y} \boldsymbol{e}_{z} \boldsymbol{e}_{x} \sin ^{2} \frac{\xi}{2} \\
= & \boldsymbol{e}_{x} \cos ^{2} \frac{\xi}{2}-\boldsymbol{e}_{y} \boldsymbol{e}_{z} \boldsymbol{e}_{x} \cos \frac{\xi}{2} \sin \frac{\xi}{2}+\boldsymbol{e}_{x} \boldsymbol{e}_{y} \boldsymbol{e}_{z} \sin \frac{\xi}{2} \cos \frac{\xi}{2} \boldsymbol{e}_{x} \boldsymbol{e}_{x} \boldsymbol{e}_{y} \boldsymbol{e}_{z} \boldsymbol{e}_{y} \boldsymbol{e}_{z} \boldsymbol{e}_{x} \sin ^{2} \frac{\xi}{2} \\
= & \boldsymbol{e}_{x} \cos ^{2} \frac{\xi}{2}-\boldsymbol{e}_{x} \boldsymbol{e}_{y} \boldsymbol{e}_{z} \sin \frac{\xi}{2} \cos \frac{\xi}{2}+\boldsymbol{e}_{x} \boldsymbol{e}_{y} \boldsymbol{e}_{z} \sin \frac{\xi}{2} \cos \frac{\xi}{2}-\boldsymbol{e}_{y} \boldsymbol{e}_{z} \boldsymbol{e}_{y} \boldsymbol{e}_{z} \boldsymbol{e}_{x} \sin ^{2} \frac{\xi}{2} \\
= & \boldsymbol{e}_{x} \cos ^{2} \frac{\xi}{2}+\boldsymbol{e}_{x} \sin ^{2} \frac{\xi}{2}=\boldsymbol{e}_{x}
\end{aligned}
$$

\section{Appendix 5}

10.1 We will verify the rationality of Eq. (41)

Using property (b) and Eq. (39), we can obtain the CRM of $\boldsymbol{R}_{Y}$, that is

$$
\begin{gathered}
\psi\left(\boldsymbol{R}_{\boldsymbol{Y}}\right)=\psi(\boldsymbol{A}) \psi\left(\boldsymbol{R}_{\boldsymbol{s}}\right) \psi\left(\boldsymbol{A}^{+}\right)+6 \sigma^{2} \boldsymbol{I}_{M N} \\
=\psi(\boldsymbol{A}) \psi\left(\boldsymbol{R}_{\boldsymbol{s}}\right) \psi^{+}(\boldsymbol{A})+6 \sigma^{2} \boldsymbol{I}_{M N}
\end{gathered}
$$

where

$$
\boldsymbol{R}_{\boldsymbol{s}}=E\left\{\boldsymbol{S \boldsymbol { S } ^ { + }}\right\}
$$

Since $\boldsymbol{R}_{\boldsymbol{s}}$ is full rank, it is easy to obtain

$$
\operatorname{rank}\left\{\psi(\boldsymbol{A}) \psi\left(\boldsymbol{R}_{\boldsymbol{s}}\right) \psi^{+}(\boldsymbol{A})\right\}=K
$$

According to the principle of MUSIC, we have

$$
\psi^{+}(\boldsymbol{A}) \boldsymbol{U}_{\psi n}=\mathbf{0}_{2 K \times(2 M-K)}
$$

Where $\boldsymbol{U}_{\psi n} \in G_{3^{2 M \times(2 M-K)}}$ composed of the eigenvectors corresponding to the $2 M-K$ smaller eigenvalues of $\psi\left(\boldsymbol{R}_{Y}\right)$. Using the property (d), Eq. (92) is equivalent to the following equation.

$$
\boldsymbol{P}_{2 K} \boldsymbol{P}_{2 K}^{+} \psi^{+}(\boldsymbol{A}) \boldsymbol{U}_{\psi n}=\mathbf{0}_{2 K \times(2 M-K)}
$$

By means of the property (e), Eq. (93) can be further expressed as

$$
\psi^{+}(\boldsymbol{A}) \boldsymbol{P}_{2 M}^{+} \boldsymbol{P}_{2 M} \boldsymbol{U}_{\psi n}=\mathbf{0}_{2 K \times(2 M-K)}
$$

Taking the multiplication on the left by $\boldsymbol{P}_{2 K}$, we have

$$
\boldsymbol{A}^{+} \boldsymbol{U}_{\boldsymbol{Y} n}=\mathbf{0}_{K \times(2 M-K)}
$$

Where $\boldsymbol{U}_{\boldsymbol{Y} n}=\boldsymbol{P}_{2 M} \boldsymbol{U}_{\psi n} \in G_{3^{M \times(2 M-K)}}$ is composed of the eigenvectors corresponding to the $2 M-K$ smaller eigenvalues of $\boldsymbol{R}_{\boldsymbol{Y}}$. Thus, Eq. (41) holds.

\section{Acknowledgements \\ The authors would like to thank the anonymous reviewers for the} improvement of this paper.

\section{Funding}

This project was supported by the National Natural Science Foundation of China (Grant No.61302017).

\section{Authors' contributions}

Tianzhen MENG conceived the basic idea and designed the numerical simulations. Minjie WU analyzed the simulation results. Naichang YUAN refined the whole manuscript. All authors read and approved the final manuscript.

\section{Competing interests}

The authors declare that they have no competing interests.

\section{Publisher's Note}

Springer Nature remains neutral with regard to jurisdictional claims in published maps and institutional affiliations. 
Received: 31 March 2017 Accepted: 6 September 2017

Published online: 13 September 2017

\section{References}

1. Lu Gan, Xiaoyu Luo. Direction-of-arrival estimation for uncorrelated and coherent signals in the presence of multipath propagation, IET Microwaves, Antennas \& Propagation, 2013, vol. 7, no. 9, pp. 746-753, DOl: https://doi. org/10.1049/iet-map.2012.0659

2. Lars Josefsson, Patrik Persson. Conformal Array Antenna Theory and Design, IEEE Press Series on Electromagnetic Wave Theory, 2006, ISBN:978-0-47146584-3

3. Z. -S. Qi., Y., Guo., B. -H. Wang. Blind direction-of-arrival estimation algorithm for conformal array antenna with respect to polarisation diversity, IET Microwaves, Antennas \& Propagation, 2011, vol. 5, no. 4, pp. 433-442, DOl: https://doi.org/10.1049/iet-map.2010.0166

4. B. H. Wang, Y. Guo, Y. L. Wang, Y. Z. Lin. Frequency-invariant pattern synthesis of conformal array antenna with low corss-polarisation, IET Microwaves, Antennas \& Propagation, 2008, vol. 2, no. 5, pp. 442-450, ISSN: 1751-8725, DOl:https://doi.org/10.1049/iet-map:20070258

5. P. Alinezhad, S.R. Seydnejad, D. Abbasi-Moghadam, DOA estimation in conformal arrays based on the nested array principles. Digit. Signal Process. 50, 191-202 (2016). https://doi.org/10.1016/j.dsp.2015.12.009

6. L. Zou, J. Lasenby, Z. He, Pattern analysis of conformal array based on geometric algebra, IET Microwaves, Antennas \& Propagation, 2011, vol. 5, no. 10, p. 12101218, ISSN: 1751-8725, DOI: https:/doi.org/10.1049/iet-map.2010.0588

7. W.U. Minjie, Z.H.A.N.G. Xiaofa, H.U.A.N.G. Jingjian, Y.U.A.N. Naichang, DOA estimation of cylindrical conformal array based on geometric algebra. Int J Antennas Propagation 2016, 1-9 (2016). https://doi.org/10.1155/2016/7832475

8. A. Nehorai, E. Paldi, Vector-sensor array processing for electromagnetic source localization. IEEE Trans. Signal Process. 42(2), 376-398 (1994). https:// doi.org/10.1109/78.275610

9. N.L. Bihan, S. Miron, J. Mars, MUSIC algorithm for vector-sensors array using biquaternions. IEEE Trans. Signal Process. 55(9), 4523-4533 (2007). https:// doi.org/10.1109/TSP.2007.896067

10. J.F. Jiang, J.Q. Zhang, Geometric algebra of euclidean 3-space for electromagnetic vector-sensor array processing, part I: modeling. IEEE Trans. Antennas Propag. 58(12), 3961-3973 (2010). https://doi.org/10.1109/TAP. 2010.2078468

11. Venzo De Sabbata, Bidyut Kumar Datta. Geometric Algebra and Applications to Physics. CRC Press, 2007, ISBN: 978-1-58488-772-0

12. J.W. Arthur, Understanding geometric algebra for electromagnetic theory (IEEE Press, New Jersey, 2011) ISBN: 978-1-118-07854-3

13. Dietmar Hildenbrand. Foundations of geometric algebra computing, 2013, Springer, ISBN: 978-3-642-31793-4, DOl: https://doi.org/10.1007/978-3-64231794-1

14. Leo Dorst, Daniel Fontijne, Stephen Mann. Geometric Algebra for Computer Science, 2007, Morgan Kaufmann, ISBN: 978-0-12-369465-2

15. John Snygg. A New Approach to Differential Geometry Using Clifford's Geometric Algebra, Birkhäuser, 2010, New York, ISBN: 978-0-8176-8282-8

16. DESCHAMPS G. A. Techniques for handling elliptically polarized waves with special reference to antennas: part II-geometrical representation of the polarization of a plane electromagnetic wave. Proc. IRE, 1951, vol. 39, p. 540544. DOl:https://doi.org/10.1109/JRPROC.1951.233136

17. W.U. Minjie, Y.U.A.N. Naichang, DOA estimation in solving mixed noncircular and circular incident signals based on the circular array. Prog. Electromagn. Res. M 53, 141-151 (2017). https://doi.org/10.2528/ PIERM16092105

18. R. Schmidt, Multiple emitter location and signal parameter estimation. IEEE Trans. Antennas Propag. AP-34(3), 276-280. ISSN: 0018-926X (1986). https:// doi.org/10.1109/TAP.1986.1143830

19. S. Miron, N.L. Bihan, J.I. Mars, Quaternion-MUSIC for vector-sensor array processing. IEEE Trans. Signal Process. 54(4), 1218-1229 (2006). https://doi. org/10.1109/TSP.2006.870630

20. Ziyang Gao, Yong Xiao. Direction of arrival estimation for conformal arrays with diverse polarizations, $201512^{\text {th }} \mid$ IEEE International Conference on Electronic Measurement \& Instruments, pp. 439-442. DOI: https://doi.org/10. 1109/ICEMI.2015.7494229

21. P. Stoica, A. Nehorai, MUSIC, maximum likelihood, and Cramer-Rao Bound. IEEE TRANS. ACOUSTICS SPEECH SIGNAL PROCESSING. 37(5), 720-741. ISSN: 0096-3518 (1989). https://doi.org/10.1109/29.17564

\section{Submit your manuscript to a SpringerOpen ${ }^{\mathcal{O}}$ journal and benefit from:}

- Convenient online submission

- Rigorous peer review

- Open access: articles freely available online

- High visibility within the field

- Retaining the copyright to your article

Submit your next manuscript at springeropen.com 